\title{
Triplet Diphenylcarbenes Pr otected by Trifluoromethyl and Bromine Groups. A Triplet Carbene Surviving a Day in Solution at Room Temperature
}

\author{
Tetsuji Itoh,§ Yoshimaru Nakata, § Katsuyuki Hirai I* and Hideo Tomioka $† *$ \\ $\S$ Chemistry Department for Materials, Faculty of Engineering and Instrumental \\ Analysis Facilities, Life Science Research Center, Mie University, Tsu, Mie 514-8507 \\ Japan and †Department of Applied Chemistry, Aichi Institute of Technology, Toyota, \\ Aichi 470-0392 Japan
}

\section{Supporting Information}

Figure S1. Transient absorption spectra obtained in LFP of $S-1-\mathrm{N}_{2}$ (a) in degassed benzene and (b) in non-degassed benzene with a 308-nm excimer laser recorded 500 $\mu$ s after the pulse. Inset shows oscillogram traces monitored at $420 \mathrm{~nm}$.

Figure S2. Transient absorption spectra obtained in LFP of $S-\mathbf{1}-\mathrm{N}_{2}$ in degassed benzene in the presence of 1,4-cyclohexadiene with a 308-nm excimer laser recorded $500 \mathrm{~ms}$ after the pulse. Inset shows oscillogram traces monitored at $410 \mathrm{~nm}$.

Figure S3. (a) MALDI-TOF-MASS spectrum of the carbene dimers from $S-1-\mathrm{N}_{2}$. (b) Simulated spectrum for the carbene dimer $+\mathrm{H}\left(\mathrm{C}_{54} \mathrm{H}_{29} \mathrm{Br}_{4} \mathrm{~F}_{12}\right)$.

Figure S4. (a) MALDI-TOF-MASS spectrum of the carbene dimers from $U-1-\mathrm{N}_{2}$. (b) Simulated spectrum for the carbene dimer $\left(\mathrm{C}_{54} \mathrm{H}_{28} \mathrm{Br}_{4} \mathrm{~F}_{12}\right)$.

Figure S5. (a) ${ }^{1} \mathrm{H}-\mathrm{NMR}$ spectrum of the carbene dimers from $\mathrm{S}-\mathbf{1}-\mathrm{N}_{2}$.

Figure S6. (a) ${ }^{1} \mathrm{H}-\mathrm{NMR}$ spectrum of the carbene dimers from $U-\mathbf{1}-\mathrm{N}_{2}$.

Figure S7. ORTEP drawings with the atomic numbering of $S-1-\mathrm{N}_{2}$ and $U-\mathbf{1}-\mathrm{N}_{2}$. The thermal ellipsoids for the non-hydrogen atoms are drawn at $50 \%$ probability level. Selected bond distance $(\AA)$ and angles (deg):

Figure S8. Selected geometrical parameters (distance in $\AA$ and angle in degree) for (2,6-dibromophenyl)[2,6-bis(trifluoromethyl)phenyl]diazomethane $\left(U-\mathbf{1 a}-\mathrm{N}_{2}\right)$ and bis(2-bromo-6-trifluoromethylphenyl)diazomethane ( $S-\mathbf{1 a - N _ { 2 }}$ ) at B3LYP/6-31G* level of theory.

Tables. Cartesian coordinates, absolute ener gies of all calculated compounds at the B3LYP/6-31G* level of theory . 


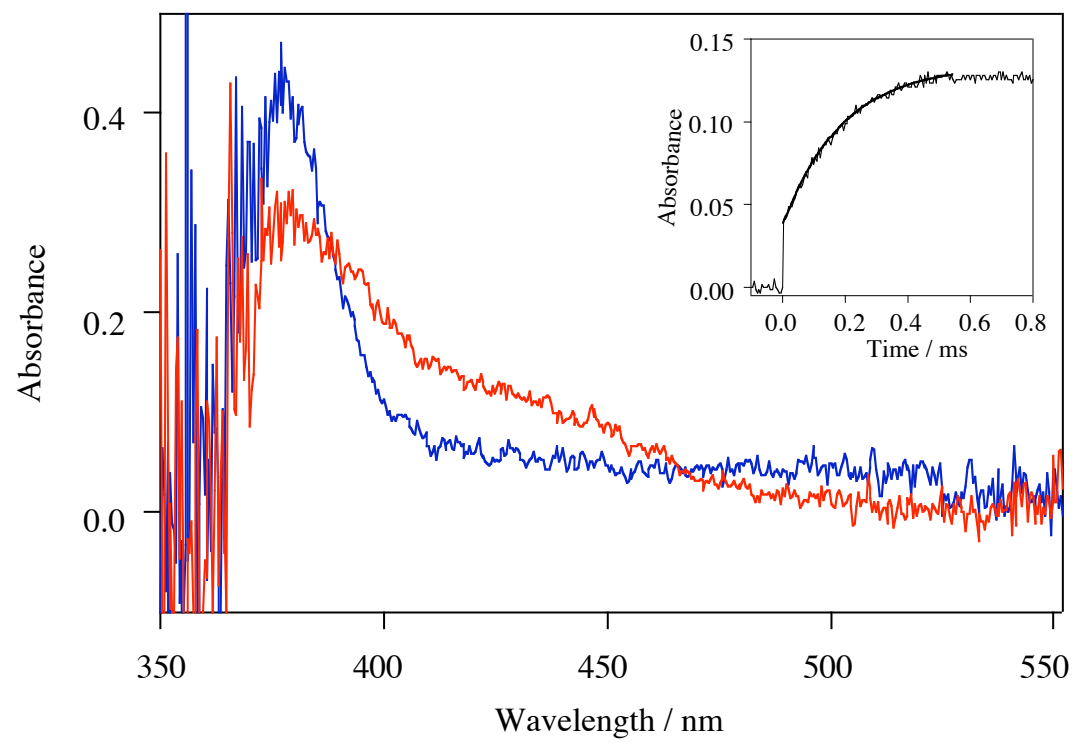

Figure S1. Transient absorption spectra obtained in LFP of $\mathrm{S}-\mathbf{1}-\mathrm{N}_{2}$ (a) in degassed benzene and (b) in nondegassed benzene with a 308-nm excimer laser recorded $500 \mu \mathrm{s}$ after the pulse. Inset shows oscillogram traces monitored at $420 \mathrm{~nm}$.

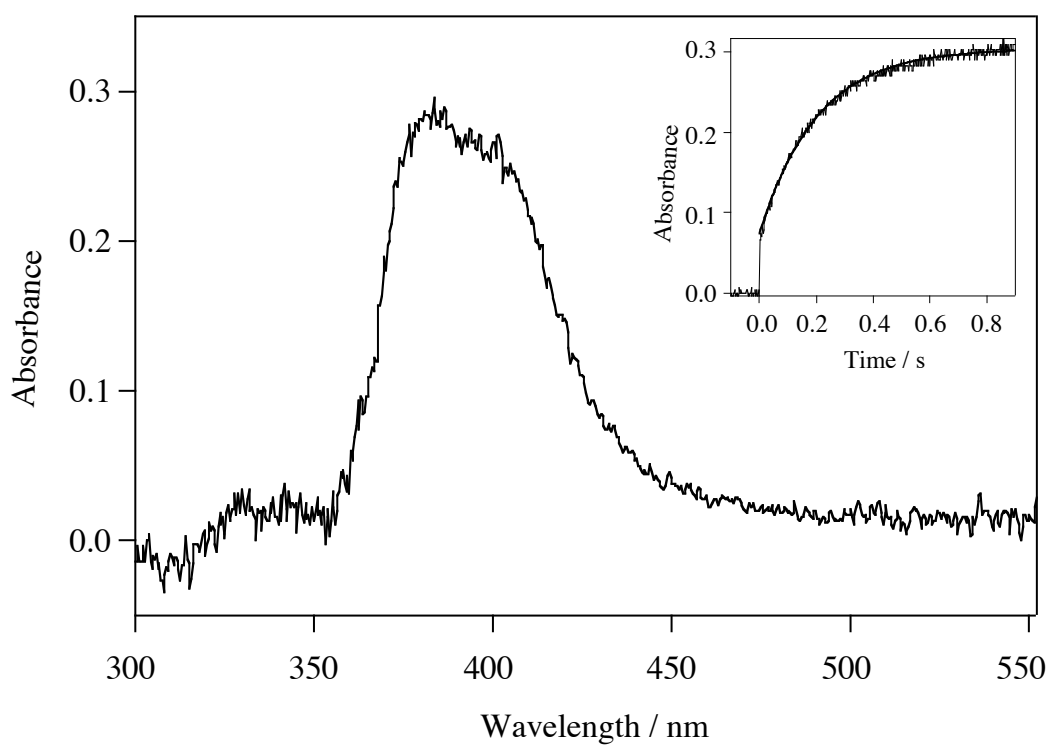

Figure S2. Transient absorption spectra obtained in LFP of $S-\mathbf{1}-\mathrm{N}_{2}$ in degassed benzene in the presence of 1,4-cyclohexadiene with a 308-nm excimer laser recorded $500 \mathrm{~ms}$ after the pulse. Inset shows oscillogram traces monitored at $410 \mathrm{~nm}$. 

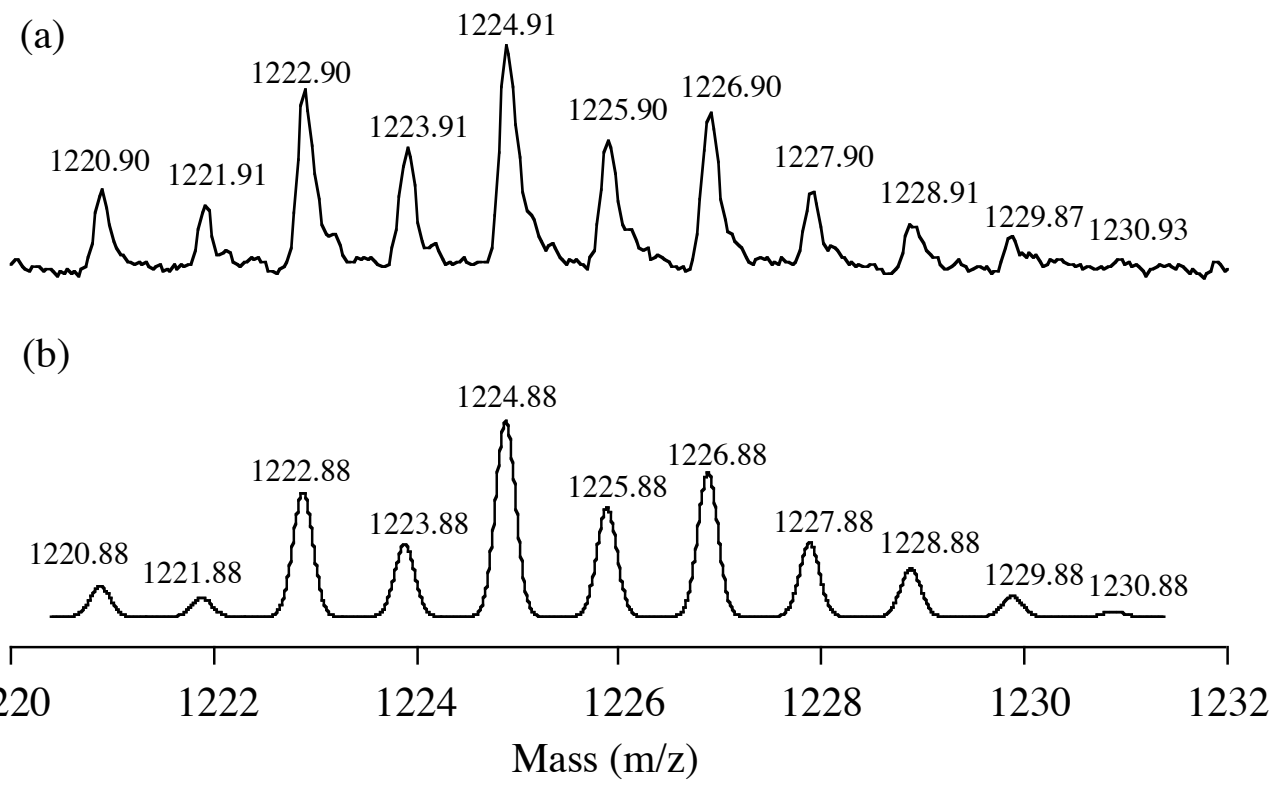

Figure S3. (a) MALDI-TOF-MASS spectrum of the carbene dimers from $S-1-\mathrm{N}_{2}$. (b) Simulated spectrum for the carbene dimer $+\mathrm{H}\left(\mathrm{C}_{54} \mathrm{H}_{29} \mathrm{Br}_{4} \mathrm{~F}_{12}\right)$.

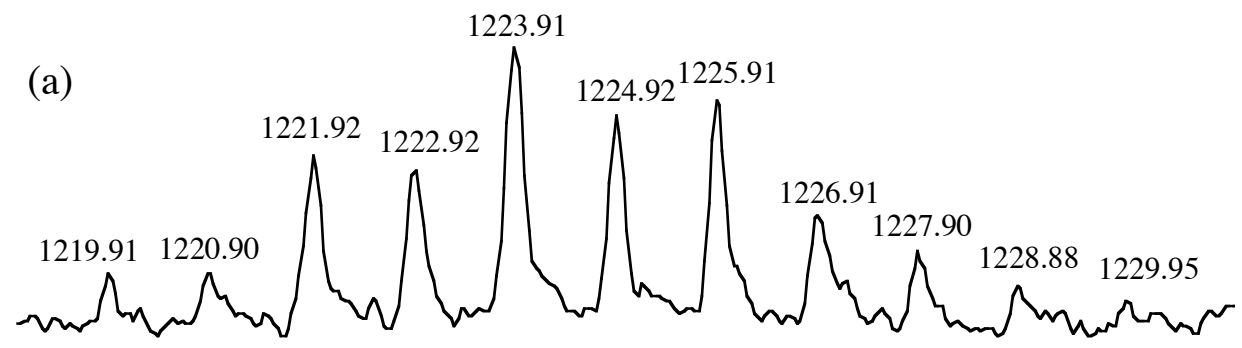

(b)

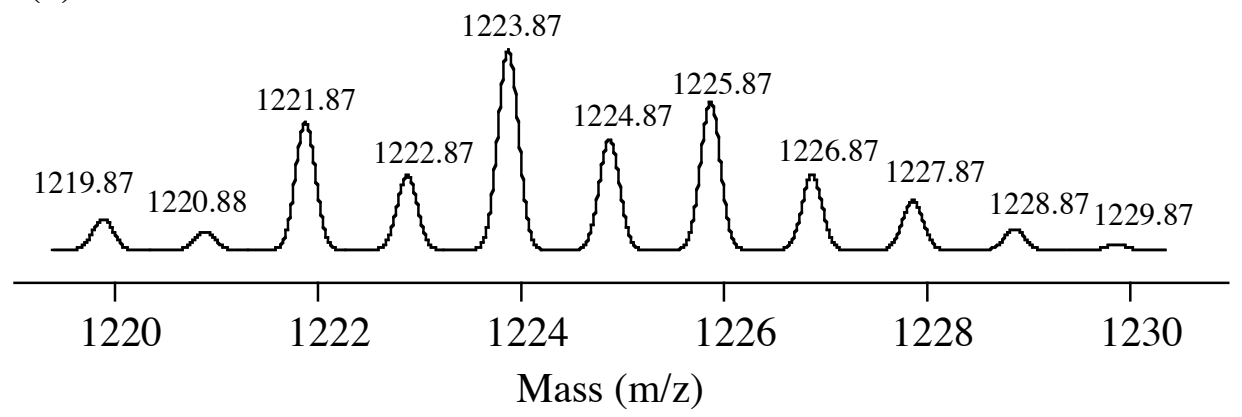

Figure S4. (a) MALDI-TOF-MASS spectrum of the carbene dimers from $U-1-N_{2}$. (b) Simulated spectrum for the carbene dimer $\left(\mathrm{C}_{54} \mathrm{H}_{28} \mathrm{Br}_{4} \mathrm{~F}_{12}\right)$. 


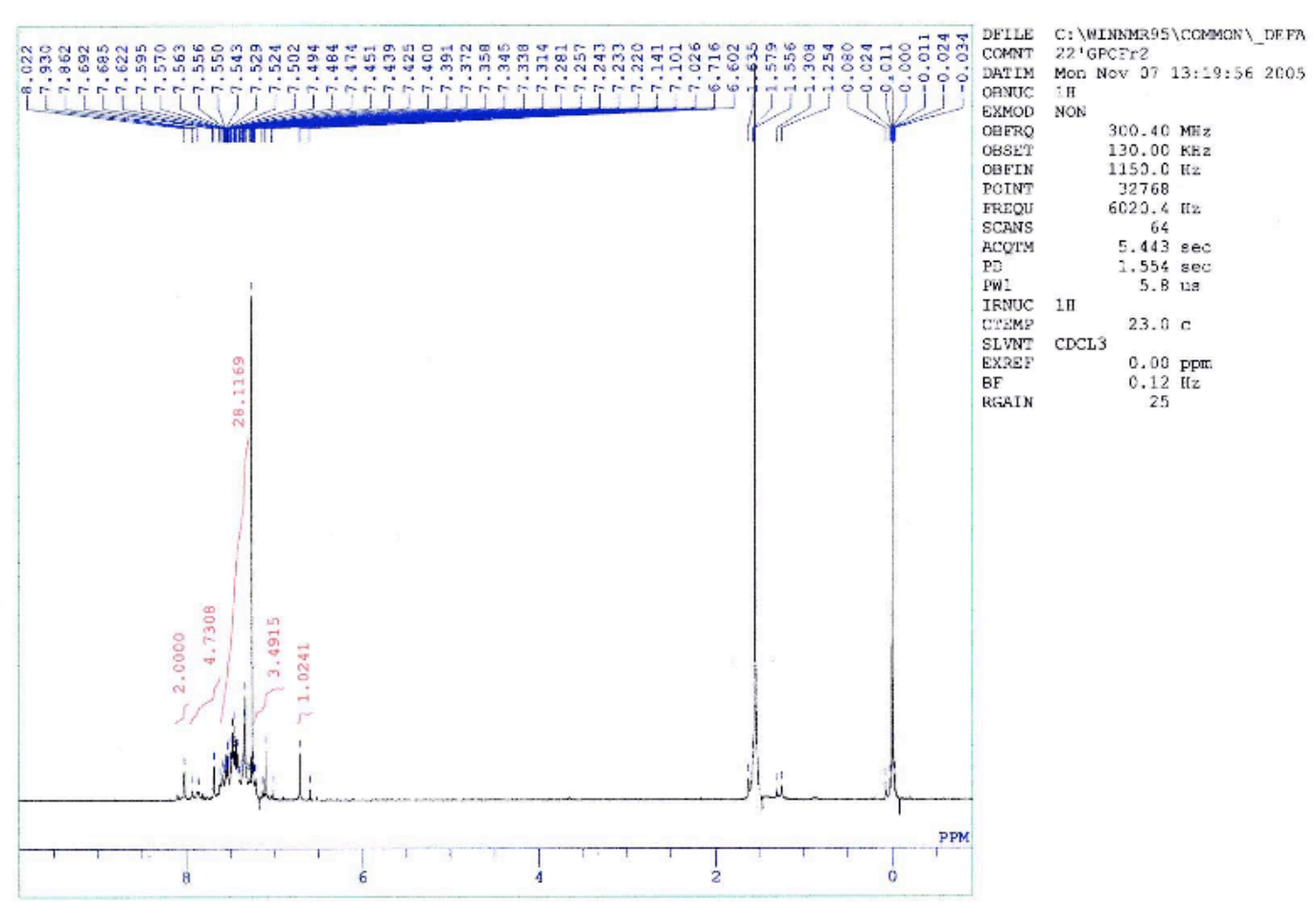

Figure S5. (a) ${ }^{1} \mathrm{H}-\mathrm{NMR}$ spectrum of the carbene dimers from $S-1-\mathrm{N}_{2}$.

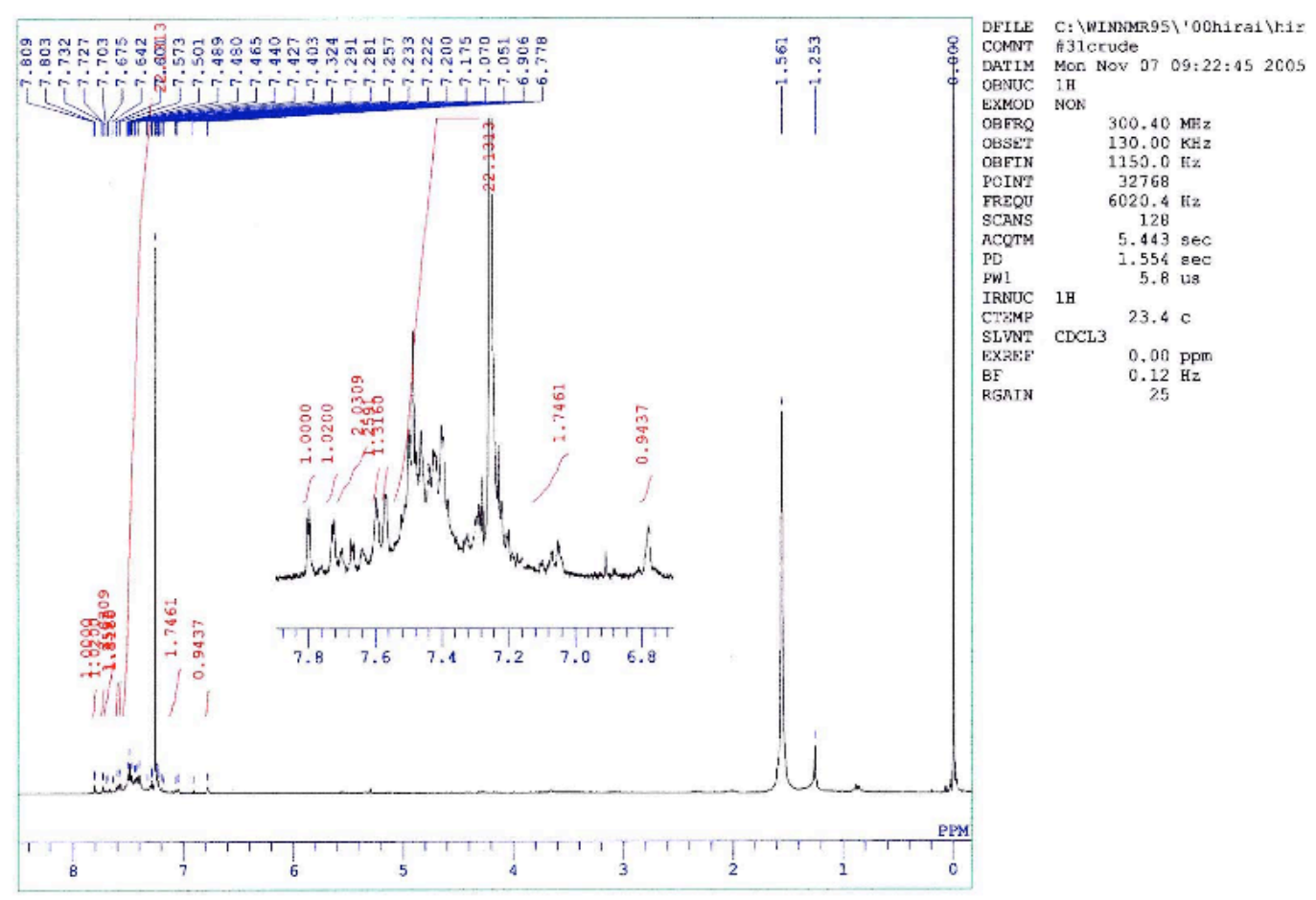

Figure S6. (a) ${ }^{1} \mathrm{H}-\mathrm{NMR}$ spectrum of the carbene dimers from $U-\mathbf{1}-\mathrm{N}_{2}$. 

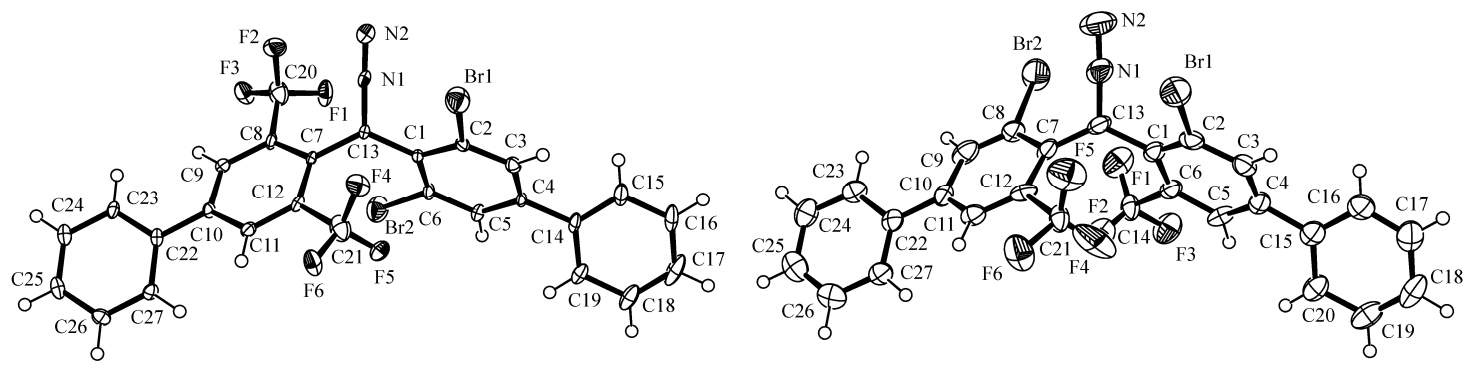

\begin{tabular}{|c|c|c|c|c|c|c|}
\hline \multicolumn{2}{|c|}{$U-1-\mathrm{N}_{2}$} & \multicolumn{5}{|c|}{$S-\mathbf{1}-\mathrm{N}_{2}$} \\
\hline & $\begin{array}{c}\theta \\
\text { Ar-C-Ar' } \\
\left.{ }^{\circ}\right]\end{array}$ & $\begin{array}{c}\text { Dihedral angle } \omega \\
{\left[^{\circ}\right]}\end{array}$ & $\begin{array}{c}\mathrm{d}_{1} \\
\mathrm{Ar}-\mathrm{C} \\
{[\AA]}\end{array}$ & $\begin{array}{c}\mathrm{d}_{2} \\
\mathrm{C}-\mathrm{A} \mathrm{r}^{\prime} \\
{[\AA]}\end{array}$ & $\begin{array}{c}\mathrm{d}_{3} \\
\mathrm{C}=\mathrm{N}_{2} \\
{[\AA]}\end{array}$ & $\begin{array}{c}\mathrm{d}_{4} \\
\mathrm{~N}=\mathrm{N} \\
{[\AA]}\end{array}$ \\
\hline$U-1-\mathrm{N}_{2}$ & $128.3(6)$ & $83.9(6)$ & $1.477(8)^{*}$ & $1.473(8)^{*}$ & $1.33(1)$ & $1.13(1)$ \\
\hline$S-1-\mathrm{N}_{2}$ & $128.7(7)$ & $78.7(8)$ & $1.45(1)$ & $1.50(1)$ & $1.33(1)$ & $1.16(1)$ \\
\hline
\end{tabular}

Figure S7. ORTEP drawings with the atomic numbering of $S-\mathbf{1}-\mathrm{N}_{2}$ and $U-\mathbf{1}-\mathrm{N}_{2}$. The thermal ellipsoids for the non-hydrogen atoms are drawn at $50 \%$ probability level. Selected bond distance $(\AA)$ and angles (deg).

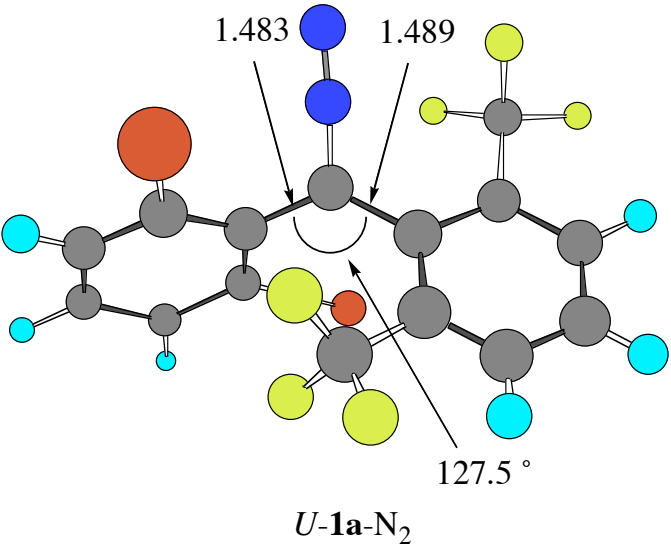

dihedral angle $70.3^{\circ}$

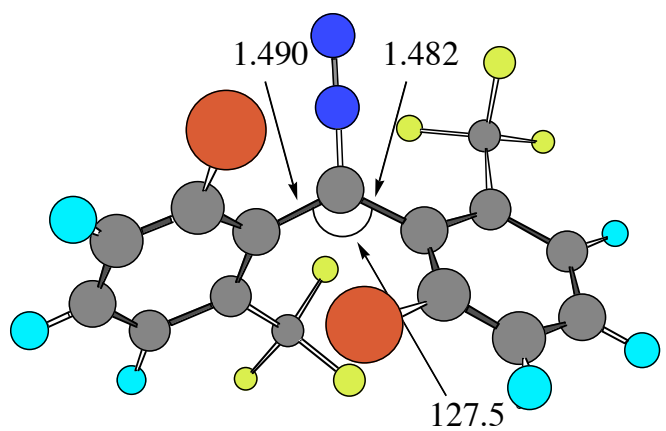

$S-1 \mathbf{a}-\mathrm{N}_{2}$

dihedral angle $70.7^{\circ}$

Figure S8. Selected geometrical parameters (distance in $\AA$ and angle in degree) for (2,6-dibromophenyl)[2,6- bis(trifluoromethyl)phenyl]diazomethane $\left(U-\mathbf{1 a}-\mathrm{N}_{2}\right)$ and bis(2-bromo-6-trifluoromethylphenyl)diazomethane $\left(S-\mathbf{1 a}-\mathrm{N}_{2}\right)$ at B3LYP/6-31G* level of theory.

Tables. Cartesian coordinates, absolute energies of all calculated compounds at the B3LYP/6-31G* level of theory

triplet bis(2,6-dibr omophenyl)carbene ( $\left.{ }^{3} 2 a\right)$ 


\begin{tabular}{|c|c|c|c|}
\hline $\begin{array}{l}\text { Symmet } \\
\text { Triplet s } \\
\text { Zero-po } \\
\text { Sum of } \\
\text { HF }=-10\end{array}$ & $\begin{array}{l}\text { ry } \mathrm{C} 1 \\
\text { tate } \\
\text { int correctior } \\
\text { electronic an } \\
785.7187057\end{array}$ & $\begin{array}{l}n=0.143351 \\
\text { id zero-point } \\
7\end{array}$ & $\begin{array}{l}(\text { Hartree/Particle }) \\
\text { Ener gies }=-10785.5753\end{array}$ \\
\hline Atomic & Coor & rdinates (Ang & gstroms) \\
\hline Type & $X$ & $\mathrm{Y}$ & \\
\hline 6 & -3.702851 & 0.698458 & -0.127453 \\
\hline 6 & -4.114298 & -0.396660 & 0.640731 \\
\hline 6 & -3.167950 & -1.262683 & 1.198385 \\
\hline 6 & -1.817356 & -1.038322 & 0.988695 \\
\hline 6 & -1.338134 & 0.059758 & 0.201165 \\
\hline 6 & -2.353012 & 0.916911 & -0.335455 \\
\hline 6 & 0.000066 & $0.339808 \quad c$ & 0.000077 \\
\hline 6 & 1.338149 & 0.059330 & $-0.201 \quad 153$ \\
\hline 6 & 1.816905 & $-1.038929-$ & -0.988773 \\
\hline 6 & 3.167380 & $-1.263737-$ & -1.198659 \\
\hline 6 & 4.114101 & -0.398030 & -0.641119 \\
\hline 6 & 3.703131 & 0.697190 & 0.127160 \\
\hline 6 & 2.353392 & 0.916108 & 0.335361 \\
\hline 35 & -1.795469 & 2.414878 & -1.368697 \\
\hline 35 & -0.559730 & -2.230361 & 1.770241 \\
\hline 35 & 0.558810 & -2.230594 & -1.770130 \\
\hline 35 & 1.796489 & 2.414173 & 1.368799 \\
\hline 1 & 4.430526 & 1.375293 & 0.559318 \\
\hline 1 & 5.172042 & -0.574581 & -0.808675 \\
\hline 1 & -3.480713 & -2.111060 & 1.796906 \\
\hline 1 & -5.172317 & -0.572866 & 0.808160 \\
\hline 1 & 3.479782 & -2.112209 & -1.797229 \\
\hline 1 & -4.429929 & $1.376843-$ & -0.559691 \\
\hline
\end{tabular}

triplet (2,6-dibromophenyl) [2,6-bis(trifluor omethyl)phenyl]carbene $\left(U-{ }^{3} 1\right.$ a)

Symmetry C1

Triplet state

Zero-point correction $=0.172768$ ( Hartree/Particle $)$

Sum of electronic and zero-point Ener gies $=-6317.407611$

$\mathrm{HF}=-6317.5803789$

\begin{tabular}{llll} 
Atomic & \multicolumn{3}{c}{ Coordinates (Angstroms) } \\
Type & $\mathrm{X}$ & $\mathrm{Y}$ & $\mathrm{Z}$ \\
- & &
\end{tabular}




$\begin{array}{lccc}6 & 3.991467 & -0.467601 & 1.225430 \\ 6 & 2.895002 & -0.525943 & 2.091350 \\ 6 & 1.601457 & -0.386516 & 1.612461 \\ 6 & 1.352469 & -0.175402 & 0.209087 \\ 6 & 2.504469 & -0.114708 & -0.650175 \\ 6 & 0.082402 & -0.054422 & -0.307773 \\ 6 & -1.284410 & 0.137613 & -0.332123 \\ 6 & -1.872352 & 1.438332 & -0.216651 \\ 6 & -3.238998 & 1.640378 & -0.300159 \\ 6 & -4.089337 & 0.548601 & -0.508664 \\ 6 & -3.567750 & -0.744591 & -0.627217 \\ 6 & -2.200541 & -0.941418 & -0.542177 \\ 6 & 2.301234 & 0.129185 & -2.124299 \\ 9 & 3.476256 & 0.183033 & -2.789737 \\ 9 & 1.659919 & 1.297114 & -2.352413 \\ 9 & 1.562946 & -0.846003 & -2.698669 \\ 6 & 0.439210 & -0.445117 & 2.570009 \\ 9 & -0.426445 & -1.434903 & 2.252202 \\ 9 & -0.266250 & 0.710026 & 2.571209 \\ 9 & 0.842798 & -0.663818 & 3.839949 \\ 35 & -0.721604 & 2.921644 & 0.072561 \\ 35 & -1.492565 & -2.698095 & -0.687775 \\ 1 & -4.223613 & -1.594161 & -0.781524 \\ 1 & -5.161250 & 0.705866 & -0.577895 \\ 1 & 3.049933 & -0.681886 & 3.152898 \\ 1 & 4.998223 & -0.580278 & 1.614079 \\ 1 & -3.639521 & 2.643286 & -0.202408 \\ 1 & 4.631431 & -0.204728 & -0.817488 \\ ----------------------------------------------------------\end{array}$

triplet bis(2-bromo-6-trifluor omethylphenyl) carbene $\left(S-{ }^{3} 1 a\right)$

Symmetry C1

Triplet state

Zero-point correction $=0.172718$ ( Hartree/Particle)

Sum of electronic and zero-point Ener gies $=-6317.407905$

$\mathrm{HF}=-6317.580623$

\begin{tabular}{lrrr} 
Atomic & \multicolumn{3}{c}{ Coordinates (Angstroms) } \\
Type & $\mathrm{X}$ & $\mathrm{Y}$ & $\mathrm{Z}$ \\
\hline----------------------------------- \\
6 & -2.366491 & 0.378670 & -0.707669 \\
6 & -3.704356 & 0.066259 & -0.536690 \\
6 & -4.071459 & -0.956607 & 0.342465 \\
6 & -3.087809 & -1.664507 & 1.044169 \\
6 & -1.745675 & -1.363393 & 0.883179 \\
6 & -1.324310 & -0.308875 & -0.005340
\end{tabular}




$\begin{array}{lccc}6 & -0.001504 & 0.003852 & -0.214613 \\ 6 & 1.324093 & 0.310963 & -0.013769 \\ 6 & 2.359798 & -0.383679 & -0.718435 \\ 6 & 3.699908 & -0.077338 & -0.553650 \\ 6 & 4.075568 & 0.946360 & 0.320857 \\ 6 & 3.098252 & 1.661752 & 1.023806 \\ 6 & 1.754016 & 1.367163 & 0.868435 \\ 35 & 1.860010 & -1.782742 & -1.901629 \\ 6 & 0.718536 & 2.167855 & 1.616848 \\ 35 & -1.878064 & 1.776613 & -1.896979 \\ 6 & -0.703492 & -2.158258 & 1.628449 \\ 1 & -4.457365 & 0.621533 & -1.085362 \\ 1 & -5.119678 & -1.203033 & 0.477834 \\ 1 & -3.372255 & -2.461379 & 1.721265 \\ 1 & 4.447963 & -0.638071 & -1.103538 \\ 1 & 5.125551 & 1.187837 & 0.451399 \\ 1 & 3.389264 & 2.459802 & 1.696710 \\ 9 & -0.143985 & 2.780728 & 0.773660 \\ 9 & 1.282699 & 3.131026 & 2.377411 \\ 9 & -0.022368 & 1.393501 & 2.440329 \\ 9 & 0.048248 & -1.377325 & 2.435627 \\ 9 & 0.148295 & -2.781419 & 0.781737 \\ 9 & -1.260895 & -3.112456 & 2.405093 \\ -----------------------------------------------------\end{array}$

\section{(2,6-dibromophenyl)[2,6-bis(trifluor omethyl)phenyl]diazomethane ( $\left.U-1 \mathrm{a}-\mathrm{N}_{2}\right)$}

Symmetry C1

Singlet state

Zero-point correction $=0.185413$ ( Hartree/Particle)

Sum of electronic and zero-point Ener gies $=-6426.931129$

$\mathrm{HF}=-6427.1165417$

\begin{tabular}{lrcc} 
Atomic & \multicolumn{3}{c}{ Coordinates (Angstroms) } \\
Type & $\mathrm{X}$ & $\mathrm{Y}$ & $\mathrm{Z}$ \\
\hline--------------- & \\
6 & -2.039014 & -1.524260 & 2.352389 \\
6 & -3.332870 & -1.479208 & 1.849452 \\
6 & -3.535994 & -1.052670 & 0.543391 \\
6 & -2.462586 & -0.656012 & -0.257340 \\
6 & -1.128781 & -0.711728 & 0.226353 \\
6 & -0.947369 & -1.164796 & 1.558834 \\
6 & 0.031896 & -0.315143 & -0.617695 \\
6 & 1.105820 & 0.650215 & -0.279374 \\
6 & 0.821004 & 1.932302 & 0.243275 \\
6 & 1.814576 & 2.855160 & 0.552929 \\
6 & 3.149682 & 2.538698 & 0.314782
\end{tabular}




\begin{tabular}{llll}
6 & 3.481564 & 1.296405 & -0.215036 \\
6 & 2.476396 & 0.371512 & -0.489395 \\
6 & 0.420241 & -1.293884 & $2.19931 \quad 1$ \\
9 & 0.363391 & -2.010583 & 3.345442 \\
9 & 0.944899 & -0.091059 & 2.528624 \\
9 & 1.307461 & -1.918977 & 1.399423 \\
35 & 3.052017 & -1.327935 & -1.145067 \\
6 & -2.823068 & -0.176366 & -1.652375 \\
9 & -4.123292 & 0.185805 & -1.724740 \\
9 & -2.104227 & 0.888430 & -2.058339 \\
9 & -2.646522 & -1.156099 & -2.572967 \\
35 & -0.977860 & 2.488874 & 0.559130 \\
7 & 0.100254 & -0.834978 & -1.821138 \\
7 & 0.205246 & -1.279682 & -2.865635 \\
1 & 4.515055 & 1.027381 & -0.400369 \\
1 & 3.929063 & 3.259075 & 0.544092 \\
1 & 1.535372 & 3.819770 & 0.960775 \\
1 & -1.863036 & -1.864087 & 3.365419 \\
1 & -4.174159 & -1.772168 & 2.469311 \\
1 & -4.540662 & -0.999196 & 0.141984 \\
\hline
\end{tabular}

bis(2-bromo-6-trifluor omethylphenyl)diazomethane ( $\left.S-1 \mathrm{a}-\mathrm{N}_{2}\right)$

Symmetry C1

Singlet state

Zero-point correction $=0.185462($ Hartree/Particle $)$

Sum of electronic and zero-point Ener gies $=-6426.931035$

$\mathrm{HF}=-6427.1164973$

\begin{tabular}{|c|c|c|c|}
\hline \multirow{2}{*}{$\begin{array}{l}\text { Atomic } \\
\text { Type }\end{array}$} & \multicolumn{3}{|c|}{ Coordinates (Angstroms) } \\
\hline & $\mathrm{X}$ & $\mathrm{Y}$ & $\mathrm{Z}$ \\
\hline 6 & 2.130192 & 2.744670 & -0.537730 \\
\hline 6 & 3.395219 & 2.191422 & -0.715165 \\
\hline 6 & 3.531349 & 0.811884 & -0.805677 \\
\hline 6 & 2.402015 & 0.001630 & -0.714955 \\
\hline 6 & 1.097069 & 0.522516 & -0.574461 \\
\hline 6 & 0.994053 & 1.935985 & -0.483164 \\
\hline 6 & -0.085989 & -0.383811 & -0.572883 \\
\hline 6 & -1.170349 & -0.451624 & 0.435238 \\
\hline 6 & -0.853143 & -0.456826 & 1.811463 \\
\hline 6 & -1.824371 & -0.450283 & 2.808549 \\
\hline 6 & -3.169202 & -0.481048 & 2.460207 \\
\hline 6 & -3.525125 & -0.526573 & 1.116487 \\
\hline 6 & -2.554948 & -0.501865 & 0.112135 \\
\hline 6 & -0.333313 & 2.638877 & -0.291211 \\
\hline 9 & -0.818804 & 2.460481 & 0.960200 \\
\hline
\end{tabular}




\begin{tabular}{llll}
9 & -1.276540 & 2.210620 & -1.152318 \\
9 & -0.220878 & 3.974052 & -0.471011 \\
6 & -3.076381 & -0.635758 & -1.307181 \\
9 & -2.428507 & 0.124833 & -2.209047 \\
9 & -4.383196 & -0.303904 & -1.387806 \\
9 & -2.976205 & -1.921930 & -1.731783 \\
35 & 2.711013 & -1.880886 & -0.687038 \\
35 & 0.959776 & -0.525218 & 2.406901 \\
7 & -0.141385 & -1.210940 & -1.590159 \\
7 & -0.152238 & -1.924501 & -2.478848 \\
1 & -4.570167 & -0.567117 & 0.836001 \\
1 & -3.933534 & -0.483713 & 3.230975 \\
1 & 4.506941 & 0.352848 & -0.919154 \\
1 & 4.270385 & 2.831235 & -0.771598 \\
1 & -1.517073 & -0.442668 & 3.848307 \\
1 & 2.015898 & 3.818583 & -0.460707 \\
\hline
\end{tabular}

\section{Complete Ref. 23}

Frisch, M. J.; Trucks, G. W.; Schlegel, H. B.; Gill, P. M.; John son, B. G.; Robb, M. A.; Cheeseman, J. R.; Keith, T.; Petersson, G. A.; Montgomery, J. A.; Raghavachari, K.; Al-Laham, M. A.; Zakrzewski, V. G.; Ortiz, J. V.; Foresman, J. B.; Cioslowski, J.; Stefanov, B. B.; Nanayakkara, A.; Challacombe, M.; Peng, C. Y.; Ayala, P. Y.; Chen, W.; Wong, M. W.; Andres, J. L.; Replogle, E. S.; Gomperts, R.; Martin, R. L.; Fox, D. J.; Binkley, J. S.; DeFrees, D. J.; Baker, J.; Stewart, J. J. P.; Head-Gordon, M.; Gonzalez, C.; Pople, J. A. GAUSSIAN 94, Gaussian Inc.: Pittsbur gh, PA, 1995. 